\title{
NAAMLIJST DER LEDEN
}

\author{
VAN HET
}

INSTITUUT.

(10. APRIL 1889.)

$\rightarrow+9063+$.

Het getal contribueerende Leden bedraagt 530, waarvan in Nederland 5 Donateurs, 5 contribueerende instellingen, en 326 gewone Leden, en in de overzeesche bezittingen en koloniën 194 gewone Leden. Met 115 wetenschappelijke instellingen en vereenigingen staat het Instituut in betrekking en telt 1 Eerelid, 3 correspondeerende Leden en 30 buitenlandsche Leden.

\section{BESCHERMHEER :}

\section{Z. M. DE K $\mathbf{O} \mathbf{I} \mathbf{N}$.}

\section{BESTUUR:}

Prof. Dr. H. KERN, Voorzitter .

Jar van aftreding

в. в. KIELSTRA, Onder-Voorzitter . . . . . . . . . . . . 1890

J. H. DE GROOT, Penningmeester . . . . . . . . . . 1892

Dr. т. c. L. WIJNMAlen, Secretaris . . . . . . . . . . . 1892

Prof. Mr. P. A. vAN DER LITH . . . . . . . . . , . . 1890

Prof. G. K. NIEManN . . . . . . . . . . . . . . . . 1890

н. Ј. вооц . . , . . . . . . . . . . . . . . 1891

Prof. Dr. K. Martin . . . . . . . . . . . . . . . . 1891

Prof. Dr. G. A. WILKEN . . . . . . . . . . . . . 1892

. . . . . . . . . . . 1893

Prof. Dr. G. SCHLEgel . . . . . . . . . . . . . . 1893

D. P. VAN DER PANT . . . . . . . . . . . . . . 1893 


\section{BUITENLANDSCHE LEDEN.}

\section{Z. H. Prins Roland Bonaparte, Saint-Cloud.}

Dr. A. REINHOLD Rost (India Office

Library), Londen.

w. w. hunter, Cherwell Edge, Oxford. Prof. angelo de gubernatis, Florence. T. J. hovell thurlow, Londen. Prof. Dr. albrecht weber, Berlijn. ALFRED VON KREMER, Weenen.

Prof. emilio teza, Piza. GUIDO CORA, Turijn.

Colonel н. YULe, Londen.

Dr. FRIEDRICH MÜLLER, Weenen.

Prof. georg gerland, Strassburg i/E. Graaf G. H. J. Meiners D'Estrey, Parijs,

4 Place St. Michel.

Mrs. De croizier, Parijs, 9 Rue du quatre Septembre.

Radja SOURINDro moHUn tagore, Mus. Doc., Calcutta.

Dr. A. B. MEIJER, Dresden.

G. DORIa, Genua.

ARISTIDE Marre, Parijs, 49, Avenue de la Grande-Armée.
Prof. Dr. G. voN DER Gabelentz, Leipzig.

Prof. Dr. E. H. giglioli, Florence.

Prof. Dr. a. Bastian, Berlijn.

Prof. Dr. R. BRAndstettek, Lucern.

Prof. Dr. Jos. KoHLer, Berlin, Landgrafenstrasse 4 .

Jules sIlvestre, Rochefort sur ner, 2 Rue des Fonderies (Charente inférieure) Frankrijk.

Prof. Ferd. BLUmentritt, Leitmeritz (Bohemen.)

x. BRAU DE SAINT POL LIAS, Parijs, 47 rue de Passy.

J. w. Maxwell, Penang, Straits Settlements.

Dr. IgNaz goLdzIHER, VII Holló-gasse 4, Budapest.

Prof. Dr. F. von RICHTHOFEN, Berlijn (Kurfürstenstr. 117).

Prof. Dr. EDWARD B. TYLOR, Oxford (University-Museum).

\section{NE D E R L A N D.}

\section{DONATEURS.}

De Nederlandsche Handelmaatschappij. I. D. pRANSEN VAN DE PUTTE, 's Hage. Mr. w. Baron van goltstein, 's Hage. Dr. a. vrolik, Arnhem. P. 's ЈАСоB, Utrecht.

\section{EERELEDEN.}

Prof. Dr. P. J. veTh, Arnhem.

\section{CONTRIBUEERENDE INSTELLINGEN.}

De Bibliotheek van de Universiteit van Amsterdam. Het Nederlandsch Bijbelgenootschap, te Amsterdam. Het Nederlandsch Zendelinggenootschap, te Rotterdam. Het Rijks Ethnographisch Museum te Leiden. Vereeniging Pomona, te Menado. 


\section{GEWONE LEDEN.}

Dr. A. т. vAN AKEN, 's Gravenhage. D. AITTON, 's Gravenhage.

Jhr. Mr. J. P. ALEwiJn, 's Gravenbage. Jhr. D. P. van alpHEN, Haarlem. Jhr. E. T. M. vaN ALPHEN, 's Gravenhage. J. H. VAN BALEN, Voorburg. Mr. c. BAKE, 's Gravenhage. Dr. H. BAVINCK, Kampen. Mr. W H. DE BEAUfort, Leusden. Jhr. Mr. K. A. GODIN DE BEAUPCRT, 's Gravenhage.

Prof. Mr. a. Beaujon, Amsterdam. G. W. BEEGER, 's Gravenhage.

Mr. L. W. c. VAN DEN BERG, Delft. Mr. w. B. Bergsma, Apeldoorn. Mr. G. H. BETZ, 's Gravenhage. P. BEIJERINCK, Leiden.

J. L. BeiJers, Amsterdam.

A. A. Bienfait, Amsterdam. Mr. A. J. E. A. BIK, 's Gravenhage. E. F. T. BIK, 's Gravenhage.

J. w. BINKEs, 's Gravenhage.

G. BIRNIE, Deventer

Dr. H. BLINK, Amsterdam. P. A. M. Boele van hensbroek, 's Hage J. BorssevaIN, Amsterdam. CH. Borssevain, Amsterdam. H. J. BOOL, Leiden.

Jhr. Mr. J. W. G. BOREEL VAN HOGELANDEN, te Velzen.

P. R. Bos, Groningen.

H. G. Boumeester, 's Gravenhage.

Mr. L. de fillietetaz bousquet, 's Hage. J. VAN BREDA DE hAAN, Leiden.

Mr. W. à BRAKEL REIGER, 's Hage. W. G. $B^{\text {on }}$ BRANTSEN VAN DE ZIJP, 's Hage. Jhr. Mr. W. M. DE BRauW, Middelburg Prof. Dr. J. TEN BRINK, Leiden. J. A. VAN DEN BROEK, Delft.
W. D. J. BROUWER, Zwolle. J. F. D. BRUNAsma, 's Gravenhage.

G. Brenner, Amsterdam.

A. WERUMEUS BUNING, Rotterdam.

J. H. DE BUSSY, Amsterdam.

P. J. BUYSKEs, 's Gravenhage

Mr. c. E. J. Graaf van bylandt, 's Hage.

H. D. CANNE, 's Gravenhage.

H. A. N. Catenius. Breda.

Prof. Dr. D. Chantepie de la saussaye, Amsterdam.

P. N. VAN DER ChIJS, Djeddah.

J. L. CluiJssenaer, Utrecht.

W. A. COBLIJN, Nijmegen.

A. Cochius, Amsterdam.

Mr. F. B. Coninck LIEfsting, 's Hage. w. COOL, Utrecht.

Prof. Mr. P. W. A. CORT VAN DER LINDEN, Groningen.

Mr. J. Couperus, 's Gravenhage.

J. T. CREMER, Haarlem.

Mr. E. J. J. B. CREMERS, 's Gravenhage. G. C. DAUM, 's Gravenhage.

Mr. W. K. Baron van DedeM, Hoorn. I. J. DERMOUT, Scheveningen.

W J. JERX, Zr. Ms Wachtschip te Batavia.

s. vaN Deventer, 's Gravenhage.

J. S. A. VAN DISSEL, Delft.

Mr. H. van DISSEL Szn., 's Gravenhage. s. C. vaN DoEsburgh, Leiden.

Prof. Mr. H. L. DRUCKER, Groningen. L. C. DUDOK DE WIT, Breukelen. Dr. P. A. c. DUMontrer, 's Gravenhage. J. DIJK wz., 's Gravenhage.

H. DYSERINCK, 's Gravenhage.

c. A. ECKStern, 's Gravenhage.

C. P. VAN EEgHEN, Amsterdam.

Jhr. Mr. W. c. a. ELOUT van SOETERwoude, 's Hage. 
Dr. a. van DER eLST, Amsterdam. Mr. A. J. VAN EMDEN, 's Gravenhage. Mr. F. v. ENGELENbURG , 's Gravenhage. Dr. W. B. J. VAN EYK, 's Gravenhage. Mr. J. c. van EIJK, Amsterdam.

Jhr. Mr. F. J. J. van EYsinga, Leeuwarden.

IJ. PEEnstra, Amsterdam.

J. H. FERGUSON, 's Gravenhage.

G. J. PILET, Utrecht.

Mr. W. Francis, Kralingen.

P. G. GEERLING, 's Gravenhage.

w. J. GeERTSEma, Amsterdam.

W. VAN GELDER, 's Gravenhage.

Mr. J. van GenNeP, Rotterdam.

Jhr. Mr. W. T. GEVERS DEYNOOT Jr.,

's Gravenhage.

Mr. F. H. GöBEL, 's Gravenhage.

Prof. Dr. M. J. DE GoEJE, Leiden.

Mr. H. M. A. B ${ }^{\text {on }}$ VAN DER gOES VAN DIRXLAND, 's Gravenhage.

Jhr. Goldman, 's Gravenhage.

Prof. J. R. P. F. GONGGRIJP, Delft.

N. GRAAPLAND, Utrecht.

W. R. DE GREVE, 's Gravenhage.

CORN'. DE GROOT, 's Gravenhage.

J. H. DE GROOT, 's Gravenhage.

Dr. D. DE HAAN, Haarlem.

R. E. DE HAAN, Winterswijk.

J. нАВвемА, Nijmegen.

Mr. H. P. L. Hamelberg, Arnhem.

Mr. G. G. VAN HARENCARSPEL, 's Hage.

L. K. HARMSEN, Leiden.

D. HARTEVELT, Leiden.

Mr. A. ғ. K. HaRtogh, Amsterdam.

Dr. H. HARTOGH HeYS VAN zOUTEVEEN, Assen.

Jhr. c. HARTSEN, 's Gravenhage.

J. J. Hasselman, Tiel.

Mr. J. HEEMSKERK AZ., 's Gravenhage.

Mr. a. heEmskerk az., Amsterdam.

Mr. B. M. vltelander hein, 's Hige.
J. J. L. HELDRING, 's Gravenhage.

B. heldring, Amsterdam.

Mr. J. E. HENNY, Amsterdam.

J. K. VAN DER HEIJDEN, Bronbeek bij A rnhem.

Mr. c. J. A. HEIJDENRIJCK, 's Gravenhage. Mr. J. C. TH. HEYLIGERS, Zalt-Bommel. p. HEIJNEN, 's Gravenhage.

н. HIEBINK, Zutfen.

Mr. s. J. HINGST, 's Gravenhage.

I. J. H. GIJSBERTI HODENPIJL, Utrecht.

P. $B^{\text {on }}$ VAN HOGENDORP, 's Gravenhage.

J. A. HOOZE, Luik.

Jhr. H. W. p. HORA siccama, 's Hage.

Dr. R. HORst, Leiden.

P. J. van houten, 's Gravenhage.

Dr. м. тн. нобтsмa, Leiden.

J. HUdig Dz., Amsterdam.

н. с. HUMME, 's Gravenhage.

Jhr. Mr. J. HUYDECOPER VAN MAARSSEveEN, Maarsseveen.

P. C. HUIJSER, 's Gravenhage.

H. A. INSINGer, Vuursche.

Mr. є. н. 's ЈАСов, Utrecht.

J. W. JäGER, 's Gravenhage.

P. W. JANssen, Amsterdam.

Dr. c. w. Janssen, Amsterdam.

C. A. J. L. JEEKEL, Huize Wijnbergen, Olst.

Dr. F. A. Jentink, Leiden.

Mr. D. Josephus Jitta, Amsterdam.

Prof. Dr. c. M. KAN, Amsterdam.

Jhr. Mr. A. P. c. van KARNEBEEK, 's Hage.

Dr. H. F. C. ten kate Jr., 's Hage.

Prof. Dr. H. KERN, Leiden.

c. E. TAN Kesteren, Brussel.

Mr. L. w. c. KeUchenius, 's Hage.

A. Н. КІЕНL, 's Gravenhage.

E. B. KIELSTRA, 's Gravenhage.

Mr. T. H. DER KINDEREN, 's Hage.

H. C. KLINKERT, Leiden.

Mr. тH. A. кLINкHA MER, 's Gravenhage. 
j. C. DE KOCK van LEEUWEN, 's Hage. A. M. Kollewisn Nz., Amersfoort.

J. KREBMER, 's Gravenhage.

p. G. KRAMP, Amsterdam.

J. D. KRUseman, 's Gravenhage. Prof. Dr. в. P. KRULJPF, Groningen.

J. A. KRUIJT, Penang.

J. KUYPER HZN., 's Gravenhage.

A. H. KUIPERs, Haarlem.

Mr. A. c. P. LAMmers vas TOORENBURG, 4. 's Gravenhage.

8. T. LAXD, 's Gravenhage.

Mr. J. w. van lansberge, Brummen.

Dr. TH. w. vaN LTTH DE JEUDE, Leiden. Mr. H. 0. VAN DER LINDEX, Dordrecht. c. J. LEENDERTZ, Leiden.

W. H. VAN LEEUWEN, Amsterdam.

Mr. J. A. LEVY, A msterdam.

Mr. H. D. LevYssohn norman, 's Hage. J. H. LIEPTINCK, Amsterdam.

Mr. JAC. LION, 's Gravenhage.

Mr. о. Ј. н. Graaf van LIMBURG strRUM, 's Gravenhage.

Mr. A. LIND, Amsterdan.

Prof. Mr. P. A. VAN DER LITH, Leiden. Dr. D. DE LOOS, Leiden.

Prof. Mr. J. DE Louter, Utrecht. G. P. LUCARdie, 's Gravenhage. D. F. W. LUCAssEN, 's Gravenhage. Mr. R. MACALESter LOUP, 's Gravenhage. Mr. H. L. M. LUDEN, Amsterdam. Mr. J. C. DE MAREZ OYENS, 's Gravenhage. W. P. MARGADANT, 's Gravenhage. Prof. Dr. K. Martin, Leiden. Mr. M. Massink, Amsterdam. Dr. B. F. MatrHes, 's Gravenhage. Mr. p. alting mees, Amsterdam. AUGUST MRsRITZ, Amsterdam.

Dr. G. A. P. MOLENGRAapr, Utrecht. Dr. K. w. M. Montiss, Schiedam. H. MULLER SZN, Rotterdam. HENDRIK P. N. MULLER, Rotterdam. 5e Volgr. IV.
Mr. A. van naAmen van eemnes, 's Hage. Mr. 8. H. NEDERBdugH, 's Gravenhage. J. R. H, NEERVOORT VAN DE POLL, Amsterdam.

Mr. н. A, A. NEYS, 's Gravenhage.

A. D. VAN DER GoN Netsched, 's Hage.

P. м. мвтsснек, 's Gravenhage.

Prof. G. K. siemans, Delft.

J. P. NIKs, Utreeht.

Jhr. Mr. o. E. vas nisPex, 's Hage.

A. J. тH. vaN NUSEN, Zevenbergen,

M. xIJHOFp, 's Gravenhage.

Mr. o. w. star vUmax, 's Gravenhage, A. P. M. VAY OORDT, Leiden.

J. D, OORTMAN GRILIKGs, Utrecht.

Prof. Mr. J. OPPENHEIM, Groningen.

c. . PABST, 's Gravenhage.

w. PAHUd de moRtanges, Brummen.

D. P. VAN DER PANT, Leiden.

Mr. D. L. P. DE PAULY, 's Gravenhage. Prof. Dr. c. A. PEkelharing, Utrecht. M. T. H. PERELAER, 's Gravenhage.

w. L. DE PETIT, 's Gravenhage.

Prof. Mr. N. G. PIERson, Amsterdam. Prof. Dr. A. PIErson, Amsterdam.

H. D. PIERson, 's Gravenhage.

c. POENSEN, Rotterdam.

Prof. Dr. J. PIJNAPPEL GZN., Leiden.

Mr. M. J. PIJNAPPEL, Amsterdam.

c. w. PLEYTE wzx., Amsterdam.

Dr. w. PLEYTR, Leiden.

A. POMPE, Breda.

T. PRIJCE, 's Gravenhage.

a. 8. PRANSEN vaN de PUTte, Arnhem.

Jhr. Mr. J. K. W. QUARLES VAN UYFORD, 's Gravenhage.

Jhr. w. VAN RADERS, 's Gravenhage.

c. J. w. RAMANN, Amsterdam.

M. A. VAN RHEDE VAX DER KLOOT, 's Hage. Jhr. Mr. G. c. J. vaN ReENEN, 's Hage. Mr. w. J. VAN WELDEREN Baron RENGERs, Leeuwarden. 
G. C. KLERK DE REUS, 's Gravenhage. Jhr. Mr. J. C. REYNST, 's Hage.

Dr. J. G. P. RIEDEL, Utrecht.

Dr. H. M. D. VAN RIEMSDIJK, Utrecht.

Dr. w. N. DU RIEU, Leiden.

J. N. ROElants, Breda.

Jhr. Mr. J. RоËLL, 's Gravenhage.

Dr. L. W. G. DE ROO, Nijmegen.

G. Baron rosenthal, Amsterdum.

J. W. ROSKEs, Rotterdam.

Mr. J. v. J. ROOYAARDS VAN DEN HAM, Utrecht.

WILLEM RUYS, Rotterdam.

Dr. e. van RIJCKEVoRsel, Rotterdam. Mr. A. W. J. PARNCOMBE SANDERs, 's Hage. Mr. J. C. J. VAN DER SCHALK, Noordwijk. Binnen.

M. M. SCHEPMAN, Rhoon, bij Rotterdam.

Mr. W. A. A.J. B $^{\text {on }}$ SCHIMMELPENNINCK VAN DER OIJE VAN DE POLL, 'S Gravenhage. Prof. Dr. G. SCHLEgEL, Leiden.

Jhr. J. H. P. vON SCHMIDT AUP ALTENsTADT, 's Gravenhage.

J. $P$. L. SCUNEIDER, Delft.

K. L. van schouwendedrg, Haarlem.

N. D. schuUrmans, Haarlem.

Dr. J. SEMMelink, 's Gravenhage.

a. M. SERvatius, Terwolde bij Deventer.

Mr. c. J. sickesz, Huize de Cloeze, Laren.

Jhr. J. D. sIx, 's Gravenhage.

Mr. L. A. J. W. Baron SLOET VAN DE BEELE, Arnhem.

Dr. H. SMEDING, Haarlem.

н. јон. SмID, Dennenoord, Laren.

JOH. F. SNELLEMAN, Rotterdam.

Dr. c. SNOUCK HURGRONJE, Leiden.

A. SOL, 's Gravenhage.

Prof. J. SPANJAARD, Delft.

Prof. Dr. J. s. SPEIJER, Groningen.

Mr. J. P. SPRENGER vaN EIJK, 's Hage. H. T. VAN STEEDEN, 's Gravenhage. c. M. F. STOCKHAUSEN, Utrecht.

w. P. VAN STOCKUM JR., 's Gravenhage.

N. P. VAN DER STOK, Rijswijk.

(Buitengedachten).

Dr. J. A. VAN DER STOK, 's Gravenhage. P. DE STOPPELAAR, Leiden.

Mr. w. STORTENBEker Jr., 's Gravenhage. N. J. struick, 's Gravenhage.

Jhr. Mr. v. DE STUERs, 's Gravenhage. Jhr. Mr. J. E. de sturler, 's Hage. Prof. Dr. w. F. G. SURINGaR, Leiden. А. W. sisthopr, Leiden.

Mr. J. P. R. TAK VAN POORTVLIET, 's Gravenhage.

P. E. tegelberg, Amsterdam.

Jhr. Mr. c. J. DEN TEX, Amsterdam. Prof. Dr. c. P. TIELE, Leiden.

Mr. G. van tienhoven, Amsterdam.

J. \&. C. A. TIMMERMan, Amsterdam.

P. P. LAGING tOBIAS, Nijmegen.

Dr. номмо tonkes, Amsterdam.

P. W. M. TRAP, Leidén.

c. E. UHLENBECK, Willemsoord.

Mr. P. c. VALCK, Gravenhage.

Prof. Dr. J. J. P. v̇aLETON, Amersfoort.

Mr. S. A. vening MEINEsz, Rotterdam.

Mr. J. A. DE vicQ, Djeddah.

T. P. VIRULY, Leiden.

Mr. D. VISSER VAN HAZERSWOUDE,

Amsterdam.

L. DE vlaming, Haarlem.

c. A. M. vaN VLIET, 's Gravenhage.

Dr. W. r. VAN DER VLIET, Delft.

B. R. P. VAN VLIJMEN, 's Gravenhage. JOOST vaN volLENHOVEN, Rotterdam.

Mr. J. A. G. Baron DE vos vaN STEENWIJK.

Brummen.

E. J. voute, Zeist.

Prof. A. c. VREEDE, Leiden.

E. DE WAAL, 's Gravenhage.

w. A. VAN WALCHEREN, 's Gravenhage.

J. WALLAND, 's Gravenhage. 
Prof. Dr. max weber, Amsterdam, tijd.|J. Wolbers, Utrecht. in Indië.

м. J. WALLER, Amsterdam. w. p. Wolterbeek, Amsterdam. J. wüste, Amsterdam.

Mr. o. J. B. Baron van wassenaer vax CATWIJCK, 's Gravenhage.

A. E. WASZKLEWICZ, 's Gravenhage.

A. W. P. WEITZEL, 's Gravenhage.

G. P. Westerman, Amsterdam.

Mr. M. P. H. WIERcX, 's Gravenhage.

P. w. WIESEMan, 's Gravenhage.

Prof. Dr. G. A. WILKEN, Leiden.

Dr. c. WINkLer, Utrecht.

Jhr. Mr. H. C. vaN DER WIJCK, 's Hage, Jhr. Mr. H. van der WIJCK, 's Hage. Dr. тн. СH. L. WisNmalen, 's Hage. WLLEM WIJT, Rotterdam. Jон. IJKемA, 's Gravenhage.

s. B. zeverion, Amsterdam.

Mr. H. zILLESEN, 's Gravenhage.

G. E. v. L. VAN ZUYLEN, 's Gravenhage.

\section{CORRESPONDEERENDE LEDEN.}

M. L. van deventer, Prinsenhage, bij Breda.

J. C. NEURDENBURG, Rotterdam.

J. D. E. SCHмretr, Leiden.

\section{NEDERLANDSCH OOST- EN WEST-INDIË.}

\section{GEWONE LEDEN.}

Mr. J. H. ABENDANon, Lid van den Raad van Justitie, te Batavia.

J. W. H. ADÈR, Predikant te Batavia.

G. N. VAN ALPHEN DE VEER, le Luit. der Infanterie, Batavia.

H. Altmans, Aspirant-controleur toegevoegd a/d. ads. Resident van Sidajoe.

B. VAN BAAK, Resident van Djokjakarta.

L. А. вакнUIs, $l_{e}$ Luit. der Infanterie, Batavia.

c. BaUMgarten, te Tjikoppô, Buitenzorg.

Mr. G. J. A. vaN BERCKel, Lid van den Raad van Justitie te Semarang.

Mr. N. P. VAN DEN BERG, President van de Javasche Bank, Batavia.

Mr. J. H. BErgsma, Lid in den Raad van Indië, Batavia.

Dr. н. J. воELMAN, te Djokdjakarta.

w. J. возвоом, Kapitein der infanterie te Semarang.

н. ш. воsмan, Tweede onderwijzer aan de kweekschool voor inl. onderwijzers, te Amboina.

D. P. VAN BRAAM MORRIS, Gouverneur van Celebes en onderhoorigheden, te Macassar. 
Dr. J. L. a. Brandes, Ambtenaar voor de Inlandsche talen, Commissaris van het Instituut, Batavia.

J. A. H. BREIJMANN, Controleur le klasse in de onderafd. XX Kota (Singkara) Sumatra's Westkust.

J. W. VAN DEN BROEK, le Luitenant te Batavia.

R. BRoNs MIDDEL, Hoofdonderwijzer te Depok.

G. P. DE BRUYN KOPS, Controleur bij het Binnenlandsch Bestuur te Amboinia. Dr. w. BURck, Adj. Directeur van 's Lands Plantentuin, te Buitenzorg.

M. BUYs, Predikant te Bandoeng (Preanger-Regentschappen).

Dr. DAvidid R. CAPriles, Geneesheer te Curaçao.

н. м. LA CHAPELLE, Referendaris hij de Algemeene Secretarie te Buitenzorg. w. p. van charante, President van het Liefdadigheids-gesticht enz. te Depok (Buitenzorg).

Mr. J. A. VAN - DER CHIJs, Hoofdambtenaar belast met het toezicht over 't Archief, Batavia.

Mons. A. C. CLAessens, Aartsbisschop van Sirace, apostolisch vicaris en pastoor te Batavia.

Mr. J."w. тh. cohen stuart, Referendaris ter Alg. Secretarie, Batavia.

Mr. R. z. Dannenborgh, President van den landraad, te Medan.

Mr. J. A. W. van davelaAR, Leeraar in de geschiedenis, land- en volk. van N. I. aau het Gymnasium Willem III, Batavia.

K. F. Van delden-lä̈rne, Assistent-resident van Kraksaän, res. Probolinggo. J. A. VAN DELDEN, Lid van de firma Reynst \& Vinju, te Batavia.

Mr. c. T H. vaN Deventer, Advocaat te Semarang.

c. DeIJKerhofr, Luitt. Kolonel, Milit. commandant in de Wester-afdeeling van Borneo.

J. A. DEZENTJE, Landhuurder, Ampal, residentie Soerakarta.

N. J. F. DINGER, Lid van de Wees: en boedelkamer, Batavia.

s. vaN DISsEL, Inspecteur van het Inlundsch onderwijs, Batavia.

в. DOUWES DEKKER, Directeur van de N. I. Levens-verzekering maatschappij te Batavia.

H. c. DOUWES DEKKER, aspirant-Controleur, te Medan.

EUG. DUBors, Officier van Gezondheid, te Pajakombo (Padangsche Bovenlanden).

P. A. L. E. VAN DIJK, Controleur le klasse te Silindoeng, afd. Si Boga, res. Tapanoeli.

Mr. W. A. ENGELBRECHT, Vice-president van het Hooggereehtshof, te Batavia.

H. E. D. engelhaRd, Controleur le klasse, in de onderafd. Moesi Oeloe, res. Palembang.

J. ENNEN, Tweede onderwijzer aan de kweekschool, Fort de Kock.

K. P. ENGELBERT VAN BEVERYOORDE, $l_{e}$ Luitenant der Iufanterie, bij het $10 \mathrm{e}$ bat. inf. te Batavia.

Mr. J. J. c. enschedé, Advocuat, Soerabaja. 
J. PH. ERMeling, Vertegenwoordiger in Ned. Indië der Billiton-Maatschappij, Buitenzorg.

Mr. w. J. essers, Raadsheer in het Hooggerechtshof van N. I., Batavia.

p. POKKENS JR., Controleur le klasse, toegevoegd aan den Hoofdinspecteur voor de suiker en rijstenltuur, enz., te Batavia.

А. н. G. ғоккеR, President van de Factory der Ned. Handelmaatschappij, Batavia.

R. FENNEMA, Ingenieur $l_{e}$ klasse te Medan.

Mr. W. DE GELDER, Procureur-Generaal bij het Hooggerechtshof van N. I., te Batavia.

D. GERTH VAN WIJK, Leeraar in de Maleische taal aan het Gymnasium Willem III, te Batavia.

G. P. H. H. GONGGRIJP, Algemeen ontvanger, Batavia.

w. P. GRoeneveldi, Directeur van het Dep. onderwijs, eeredienst en nijverheid, Batavia.

D. GRoeneveli, Directeur der Javasche Bank, te Batavia.

A. r. P. GRAAFLAND, Controleur 2e kl. in de onderafafd. Indragiri, afd. Lingga (res. Riouw en onderhoorigheden).

D. GRIVEL, Tijd. Schoolopziener bij het Inlandsch onderwijs, te Fort de Kock. (Padangsche Bovenlanden).

Dr. J. J. M. DE GROот, Tolk voor de Chineesche taal, tijdelijk in China (Amoij).

H. J. DE GROOT, Asp. ambtenaar, Batavia.

Dr. J. Groneman, Geneesheer, te Djokjakarta.

Dr. J. G. н. GUNNING, Ambtenaar voor de Inlandsche talen, tijdelijk belast met geven van onderwijs in de Jav. taal aan het Gymn. Willem III, te Batavia.

Dr. c. GUtTELing, Eerste Stadsgeneesheer, Batavia.

Mr. J. А. налкмan, Redacteur Bat. Handelsblad, Batavia.

c. J. VAN HAASTERT, Hoofdonderwijzer aan de kweekschool te Bandoeng.

A. HAGA, Luitenant-Generaal, Commmandant van het leger en Chef van het Dep. van Oorlog, te Batavia.

Dr. B. Hagen, Geneesheer, te Laboean Deli.

G. A. HANSEN, Kapitein Inf. O. I. L., te Kota Radja.

J. P. c. hartevelt, Referendaris bij het Dep. van Financiën, te Batavia.

A. L. Van hasselt, Resident van Tapanoeli (Sumatra's Westkust), Batavia.

o. L. Helprich, Controleur 2e klasse in de afd. Mana en Pasoemah Oeloe Mana, res. Benkoelen.

J. vAN HENGEL, Controleur 2e klasse, te Indrapoera (Sumatra's Westkust).

J. heiJting, Resident der Preanger-Regentschappen, Bandoeng.

D. HEIJTINg, Resident van Amboina.

H. A. HiJMans van anRooy, Secretaris ter Oostkust van Sumatra, te Medan.

B. новтіnк, Tolk voor de Chineesche taal te Deli Medan (Sumatra's Oostkust). 
G. w. w. c. Baron van HoËvelL, Assistent-resident van Gorontalo (Menado).

w. c. Hoogkamer, Assitent-resident van de Afdeeling Tanah Datar (Fort van der Capellen.)

w. новzоо, Zendeling, Semarang.

Dr. J. w. hoprmans, Geneesheer-directeur van het krankzinigengesticht te Soerabaja.

к. ғ. ноцLе, Adviseur, Waspada (Preanger Regentschappen).

Dr. D. w. HORSt, Assistent-resident der afd. Lingga (Riouw en onderh.)

B. v. HouthuiJsen, Notaris, te Semarang.

Mr. A. J. IMminck, Raadsheer in het Hoog Gerechtshof, Batavia.

Dr. ј. к. Јасовs, Officier van Gezondheid, le Klasse, te Serang.

A. M. JoEkes, Assistent-resident te Amoentai (Z. en O. afd. van Borneo).

Mr. Dr. J. c. G. Jonker, Ambtenaar voor de Inlandsche talen, Macassar.

P. W. A. KERN, Asp. ambtenaar, Madioen.

L. Knappert, Aspirant-controleur in de Afd. Painan, Sumatra's Westkust.

J. Knebel, Controleur le klasse te Tjikadang in de residentie Preanger Regentschappen.

C. P. J. van Koetsveld, Assistent-resident van Koetei (Borneo.)

c. w. r. Kooman, Asp. controleur in de res. Cheriben.

P. J. Kooreman, Assistent-resident van de afd. Painan, Sumatra's Westkust.

G. J. VAN KOOTEN, Kapitein bij den generalen staf, N. I., Batavia.

Jhr. w. н. w. DE коск, Adjunct-inspecteur van Financiën, te Macassar.

J. c. Th. Kroesen, Resident van Soerahaja.

R. c. Kroesen, Gouverneur van Sumatra's Westkust, Padang.

c. A. Kroesen, Controleur le klasse in de onderafd. Boven Langkat (Bindjei), Sumatra's Oostkust.

J. A. Kroesen, Controleur in de residentie Oostkust van Sumatra, afd. Batoe Bara, Laboean Roekoe.

L. CH. DE KRUYF, Notaris te Probolinggo.

J. c. KUMMER, Adj. Inspecteur lager onderwijs, te Magelang, res. Kedoe.

c. H. M. LE ROUX, Secretaris der residentie Amboina.

K. F. H. VAN LANGen, Assistent-resident van Meester Cornelis (Batavia).

Dr. w. van LINGen, Predikant te Medan (Sumatra's Oostkust).

H. vaN LOKHORSt, Kolonel, Chef Geneesk. dienst, Batavia.

P. E. LUITJEs, Hoofdonderwijzer aan de kweekschool voor inlandsche onderwijzers te Amboina.

H. L. C. TE MECHeLen, (titulair) Resident, Rembang.

Mr. Ј. н. MEIss, Griffier bij den Landraad te Madioen.

w. MEIJER, Hoofdonderwijzer aan de school voor zonen van Inlandsche hoofden te Magelang (Kedoe.)

J. J. MEIJER, Controleur 2e klasse bij het Binnenlandsch Bestuur te Rangkas Betoeng, Bantam. 
J. E. DE MEIJIER, Ingenieur le kl. b/d Waterstaat en B. O. W. in N. I. Batavia. Mr. CH. van medrs, Griflier bij het Hof van Justitie, Commissaris voor Suriname, te Paramaribo.

L. J. J. MichIblsen, Lid in den Raad van Ned.-Indië, Batavia.

A. H. Moorrees, Controleur le klasse te Oelak Tanding (Palembang).

o. M. DE MUNNICK, Resident van Batavia.

c. VAN DER goN NEtscher, Assistent-resident van Panaroekan, Sitoebondo, Besoeki.

J. B. neumans, Controleur le klasse in de onderafdeeling Ommelanden van Padang (Loeboeh Begaloeng), Sumatra's Westkust.

w. c. NIeUWenhulJzen, Kapitein bij den Gen. staf te Batavia.

Dr. J. c. c. W. VAN NOOTEN, Leeraar in de wis- en natuurkunde aan de Hoogere Burgerschool te Soerabaja.

H. A. DE Noov, Onderwijzer te Semarang.

Mr. A. NUHOUT VAN DER VEEN, Referendaris bij het Departement van Justitie, Batavia.

Dr. H. J. ofperhaus, Predikant te Probolinggo.

c. J. okHUIJze, le Luitenant der Infanterie, te Ternate.

G. oostergetel, Hoofdonderwijzer, Bandjermasin.

c. A. van ophulJzen, Hoofdonderwijzer aan de Kweekschool te Padang Sidempoean (Tapanoeli).

T. OTTOLANDER, Sitoebondo.

J. PEelen, Adjunct-inspecteur van Financiën, Soerabaja.

H. c. G. PEeters, le luitenant der infanterie bij het 11, bat. inf. te Meester Cornelis.

G. w. H. Peltzer, Controleur le klasse in de onderafd. VIII Kota en VII Loerah, Sumatra's Westkust.

Mr. M. C. PIEPERs, Raadsheer in het Hoog-Gerechtshof, Batavia.

Jhr. F.: POMPE vaN MEERDERvoort, Luit. Kolonel bij den Gen. Staf, te Batavia.

H. E. Prins, Assistent-resident in de Afd. Batipoe en X Kota (Papang Pandjang).

F. H. J. PRIVÉ, O. I. ambtenaar in de residentie Besoeki.

A. PRUYS VAN DER hOEVEN, Lid in den Raad van Nederl. Indië, Batavia.

RADEN MAS ISMANGOEN DANOE WINOTO, Adjunct-inspecteur van het Inlandsch onderwijs, Probolinggo.

raden mas toemenggoeng pandj adiningrat, Regent van Demak.

H. L. JANSSEN vaN RAAY, Directeur van het Departement Burgerl. Openbare Werken, Batavia.

G. P- A. RENAUD, Ingenieur le klasse, te Batavia.

J. P. A. DE RoolJ, Controleur le klasse te Soeliki (Sumatra's Westkust).

в. J. E. Rовкотт, Assistent-resident van Tebing Tinggi, res. Palembang.

J. A. van rijn van aLKemade, Controleur 2e klasse te Bengkalis, Sumatra's Oostkust. 
P. J. van santen, Pnstoor te Batavia.

c. J. vaN schelLe, Ingenieur le klasse, te Muntok.

G. A scherer, Resident te Medan (Deli), Oostkust van Sumatra.

А. H. W. SCHEUER, Kapitein der Infanterie, te Macassar.

P. G. SCHMIDHAMER, le Luitenant bij bet $3^{\mathrm{e}}$ depot-bataillon der Infanterie in w. м. H. vaN schMID, Controleur le klasse B. B., Gorontalo. garnizoen te Amboina.

w. A. SCHNEIDER, Majoor bij het Garn. bat. te Atjeb en onderboorigheden.

J. G. sснот, Contrôleur te Fort de Kock.

J. A. SCHRöDER, Hoofdagent der N. I. Stoomvaartmaatschappij, Batavia.

L. P. tUiJl schuitemaker, Hoofdonderwijzer aan de kweekschool te Probolinggo.

Ј. н. . schultz, Adsistent-resident van de afdeeling Noorderdistricten van het Gouvern. Celebes en onderhoorigheden.

w. H. SENN vaN BASEL, Tjandjoer (Preanger Regentschappen).

w. p. sIKMan, Resident van Benkoelen.

g. sieburgh, Controleur der 2e klasse te Tamiang (Seroewaij), Sumatra's Oostkust.

G. $\boldsymbol{x}$. SOETERs, Kapitein bij den Generalen staf, te Batavia.

E. P. c. sol, Lid der Algemeene Rekenkamer, te Batavia.

Dr. J. H. P. SOLLEWYN GELPKe, Resident van Cheribon.

A. J. SPAAN, Resident van Soerakarta.

J. stauL, Majoor te Kota Radja (Atjeh).

J. w. stempoort, Majoor der genie te Batavia.

Dr: J. P. vAN DER STOK, Directeur van het Observatorium te Batavia.

A. P. Stoorvogre, Controleur te Ponorogo (Madioen).

p. L. K. Storm van 's Gravesande, Controleur le klasse te Laboean Deli.

J. stormer, Controleur, te Batjan.

H. N. STUART, Tolk voor de Chineesche taal, Semarang.

E. C. BARON SWEERTS DE LANDAS WIJBORgh, Eerste Gouvernements-Secretaris, Batavia.

TJOA DJIEN SING, Kapitein der Chineezen, te Soerabaja.

H. K. P. VAN TEYN, Generaal-Majoor, Civiel en militair Gouverneur van Atjeh, te Kota Radja.

J. L. VAN DER TOORN, Hoofdonderwijzer, Fort de Kock (Padangsche Bovenlanden).

Dr. M. TREUB, Directeur van 's Lands Plantentuin, te Buitenzorg.

w. L. troostenburg de bruisn, Controleur 2e klasse in de Afd. Soepajang (Koebang Doewa) Sumatra's Westkust.

s. w. TROMP, Assistent-Resident van Sintang (Westerafd. Borneo).

F. Twiss, Controleur le klasse te Loeboek Basoeng, afd. Priaman, res. Padangsche Benedenlanden.

H. J. TYDEMan, Resident van Tegal.

D. P. UHLENBECK, Referendaris ter Algemeene Secretarie, Batavia. 
H. тh. J. ujutrebroeck, 2e Onderwijzer aan de kweekschool te Probolinggo. Mr. A. w. c. verweis, President van den landraad, Batavia.

J. M. van vleuten, Directeur Dep. Binnenlandsch Bestuur, Batavia.

J. DE VOGEL, Asp. ambtenaar, Pekalongan.

L. M. voncK, Controleur 2e klasse te Sekajoe, res. Palembang.

Dr. A. G. vorderman, Stadsgeneesheer te Batavia.

M. Caesar voute, Landsadvocaat, Semarang.

w. H. VRIJBURG vaN DER HELL, Controleur, te Soekaboemi.

Mr. R. J. DE WAL, Griffier bij den Landraad te Tjengkalek, Kediri.

c. w. J. WENNEKER, Pastoor, Soerabaja.

Mr. w. A. P. p. L. WINckel, President der Landraden te Amboina, Wahaai en Saparoea, te Amboina.

M. WILLEMSTIJ, Kolonel-intendant, Batavia.

M. н. Witbols pedgen, Controleur le klasse te Siak, Sumatra's Oostkust.

Jhr. o. VAN DER WIJCK, Secretaris van het Gouvernement, Batavia.

J. w. Young, Tolk voor de Chineesche taal in de Westerafdeeling van Borneo, Singkawang.

J. W. IJZERMAN, Hoofdingenieur, Padang.

B. van zUTPHen, Resident van Bali en Lombok, Boeleleng.

A. H. E. zWAGER, Secretaris van den Raad van Indië, Commissaris van het Instituut, Batavia.

F. J. т. van zisll de Jong, Generaal-Majoor, Chef van de 2e afd. Dep. van Oorlog, Batavia. 